\title{
Star multigraphs with three vertices of maximum degree
}

\author{
By A. G. CHETWYND \\ Department of Mathematics, University of Lancaster \\ AND A. J. W. HILTON \\ Department of Mathematics, University of Reading
}

(Received 30 September 1985)

\section{Introduction}

The graphs we consider here are either simple graphs, that is they have no loops or multiple edges, or are multigraphs, that is they may have more than one edge joining a pair of vertices, but again have no loops. In particular we shall consider a special kind of multigraph, called a star-multigraph: this is a multigraph which contains a vertex $v^{*}$, called the star-centre, which is incident with each non-simple edge. An edgecolouring of a multigraph $G$ is a map $\phi: E(G) \rightarrow \mathscr{C}$, where $\mathscr{C}$ is a set of colours and $E(G)$ is the set of edges of $G$, such that no two edges receiving the same colour have a vertex in common. The chromatic index, or edge-chromatic number, $\chi^{\prime}(G)$ of $G$ is the least value of $|\mathscr{C}|$ for which an edge-colouring of $G$ exists. Generalizing a well-known theorem of Vizing [14], we showed in [6] that, for a star-multigraph $G$,

$$
\Delta(G) \leqslant \chi^{\prime}(G) \leqslant \Delta(G)+1,
$$

where $\Delta(G)$ denotes the maximum degree (that is, the maximum number of edges incident with a vertex) of $G$. Star-multigraphs for which $\chi^{\prime}(G)=\Delta(G)$ are said to be Class 1, and otherwise they are Class 2.

This paper is one of several we are writing on star-multigraphs and their applications. Star-multigraphs are turning out to be a very useful tool in the study of the chromatic index of simple graphs, as well as being of some interest in their own right. In particular, we shall use the results in this paper to obtain further results on the chromatic index in [7] and [8]; furthermore, in [11] C. A. Rodger and the second author apply results in this paper to the study of decompositions of complete graphs and to totally symmetric quasigroups.

For $n \geqslant 4$, let $K_{n}^{*}$ denote the star-multigraph obtained from $K_{n}$ by removing one edge, say $v^{*} v^{\prime}$, and doubling two other edges on $v^{*}$, say $v^{*} v_{1}$ and $v^{*} v_{2}$. For $n \geqslant 1$, let $K_{n}^{\dagger}$ denote the star multigraph obtained from $K_{n}$ by doubling one edge. The main theorem we prove in this paper is:

THEOREM 1. Let $G$ be a connected star multigraph with star-centre $v^{*}$, with three vertices of maximum degree including $v^{*}$, and with no induced subgraph on three vertices having more than $\Delta(G)$ edges. Then $G$ is Class 2 if and only if either

(1i) $G=K_{2 n+1}^{*}$ for some $n \geqslant 2$, or

(1ii) G has three vertices of degree $|V(G)|-1$ and the remainder have degree $|V(G)|-2$, 
or

(1 iii) $\Delta(G)=2 m+1 \geqslant 3$ for some integer $m, G$ contains a bridge $e$, and

where

$$
G \mid e=G_{1} \cup G_{2},
$$

and

$$
G_{1} \cap G_{2}=\varnothing, \quad \Delta\left(G_{1}\right)=2 m+1 .
$$

$$
G_{1}=K_{2 m+1}^{*} \quad(\text { for } m \geqslant 2) \text { or } G_{1}=K_{2 m+1}^{t} \text {. }
$$

Star multigraphs satisfying (1ii) must have odd order; all simple graphs satisfying (1 ii) are obtained from $K_{2 n+1}$ by removing $n-1$ independent edges.

Since no more than $\lfloor|V(G)| / 2\rfloor$ edges can have the same colour in an edge-coloured multigraph $G$, if

$$
|E(G)|>\Delta(G)\left\lfloor\frac{|V(G)|}{2}\right\rfloor
$$

then $G$ is necessarily Class 2. If $G$ satisfies this condition, then $G$ is called overfull. A very common reason for a multigraph to be Class 2 is that it contains an overfull subgraph $H$ with $\Delta(H)=\Delta(G)$. If

$$
|E(G)|=\Delta(G)\left[\frac{|V(G)|}{2}\right\rfloor+1
$$

then $G$ is called just overfull. A multigraph $G$ is critical if it is connected, Class 2 and $\chi^{\prime}(G \backslash e)<\chi^{\prime}(G)$ for all $e \in E(G)$. A result which is more or less equivalent to Theorem 1 is:

TheOREM 2. Let $G$ be a star multigraph with star-centre $v^{*}$ and with at most 3 vertices of maximum degree, including $v^{*}$. Then the following are equivalent:

(2i) $G$ is critical;

(2ii) $G$ is just overfull;

(2iii) $G$ is one of the following graphs:

(a) $|V(G)|=3$ and $|E(G)|=\Delta(G)+1 \geqslant 3$;

(b) $G=K_{2 n+1}^{+}$for some $n \geqslant 1$;

(c) $G=K_{2 n+1}^{*}$ for some $n \geqslant 2$;

(d) $|V(G)|$ is odd and $G$ has three vertices of degree $|V(G)|-1$ and the remainder have degree $|V(G)|-2$.

A conjecture formulated in [3] by Chetwynd and Hilton was:

CONJECTURE 1 . Let $1 \leqslant s \leqslant n$. Let $G$ be a regular star multigraph on $2 n+2$ vertices of degree $2 n+1-s$. Suppose $G$ does not contain a subgraph on three vertices with $2 n+2-s$ edges. Then $G$ is Class 1.

This was proved for $s=1$ or 2 in [3]. Here we show that Conjecture 1 is true for $s=3$.

THEOREm 3. Let $1 \leqslant s \leqslant 3$. Let $G$ be a regular star multigraph on $2 n+2$ vertices of degree $2 n+1-s$. Suppose $G$ does not contain a subgraph on three vertices with $2 n+2-s$ edges. Then $G$ is Class 1 .

In the case when $G$ is a simple graph, Theorem 3 was proved in [5]. Of course, a regular star multigraph of odd order is overfull and consequently Class 2.

We now turn our attention to simple graphs. We propose the following conjecture. 
CoNJECTURE 2. Let $G$ be a simple graph with $\Delta(G)>\frac{3}{10}|V(G)|$. Then $G$ is Class 2 if and only if $G$ contains an overfull subgraph $H$ with $\Delta(G)=\Delta(H)$.

Petersen's graph shows that the figure $\frac{3}{10}$ would be best possible. By applying Theorem 1, we prove Theorem 4 below, which verifies Conjecture 2 in one case. This particular case will be of great use in a forthcoming paper by C.A. Rodger and the second author on triangulating nearly complete graphs [11].

THEOREM 4. Let $G$ be a simple graph with $|V(G)|=2 n+1, \Delta(G)=2 n-2$ and

$$
|E(G)|=\Delta(G) \cdot\left\lfloor\frac{|V(G)|}{2}\right\rfloor=\left(\begin{array}{c}
2 n+1 \\
2
\end{array}\right)-3 n .
$$

Then $G$ is Class 2 if and only if $G$ contains an overfull subgraph $H$ of maximum degree $\Delta(H)=\Delta(G)$.

Analogous results were proved by Plantholt[13] for $\Delta(G)=|V(G)|-1$ and by Chetwynd and Hilton [3] for $\Delta(G)=|V(G)|-2$. Theorem 4 itself will be improved upon, partly by removing the restriction on $|E(G)|$, in [7] and [8].

\section{Preliminary results}

We now give a number of Lemmas which we shall need to use.

The first is due to Chetwynd and Yap[9], extending results of Jakobsen [12] and Beineke and Fiorini[1]. Let $P$ be the graph obtained from Petersen's graph by removing one vertex ( $P$ is an example of a critical graph which is not overfull).

Lemma 2.1. Let $G$ be a simple critical graph with $|V(G)| \leqslant 10$. If $G \neq P$, then $G$ is just overfull.

The next few lemmas are proved in [6].

LEMMA 2.2. Let $G$ be a critical star multigraph with star centre $v^{*}$, where $d\left(v^{*}\right)=\Delta(G)$, and with $r$ vertices of maximum degree. Then

$$
\Delta(G) \geqslant \begin{cases}\frac{2(|V(G)|-1)}{r} & \text { if } r \geqslant 4, \\ \frac{2|V(G)|}{3} & \text { if } r=3 .\end{cases}
$$

LEMMA 2.3. Let $G$ be a star multigraph with at most two vertices of maximum degree, and let $G$ not contain a subgraph on three vertices with $\Delta(G)+1$ edges. Then $G$ is Class 1 , unless, for some $n \geqslant 2, G=K_{2 n+1}^{\dagger}$.

LEMMA 2.4. Let $G$ be a critical star multigraph with star centre $v^{*}$, where $d\left(v^{*}\right)=\Delta(G)$, with $r$ vertices of maximum degree, and with maximum multiplicity of an edge $\mu$. Then

$$
\Delta(G) \leqslant|V(G)|+r+\mu-5 .
$$

In a star multigraph $G$, for each $v \in V(G)$, let $d^{*}(v)$ be the number of edges joining $v$ to vertices of maximum degree.

LEMMA 2.5. Let $G$ be a critical star multigraph with star centre $v^{*}$, where $d\left(v^{*}\right)=\Delta(G)$. Let $u$ and $w$ be adjacent vertices, $w \neq v^{*}$. Then

$$
d^{*}(w) \geqslant\left\{\begin{array}{lll}
\Delta(G)-d(u)+1 & \text { if } & d(u)<\Delta(G), \\
2 & \text { if } & d(u)=\Delta(G) .
\end{array}\right.
$$


LEMMA 2-6. Let $G$ be a star multigraph with star centre $v^{*}$, where $d\left(v^{*}\right)=\Delta(G)$. Let $d^{*}(w) \leqslant 1$ for some $w \in V(G) \backslash\left\{v^{*}\right\}$, let $e \in E(G)$ and let $e$ and $w$ be incident. Then

and

$$
\Delta(G)=\Delta(G \mid w) \Rightarrow \chi^{\prime}(G)=\chi^{\prime}(G \mid w)
$$

$$
\Delta(G)=\Delta(G \mid e) \Rightarrow \chi^{\prime}(G)=\chi^{\prime}(G \mid e) .
$$

LEMMA 2·7. Let $G$ be a critical star multigraph with star centre $v^{*}$, where $d\left(v^{*}\right)=\Delta(G)$, with at least three vertices of maximum degree. Then either $G$ is a simple graph of odd order which is just overfull and with $\Delta(G)=|V(G)|-1$, or there is a vertex which is non-adjacent to $v^{*}$.

LEMma 2:8. Let $G$ be a star multigraph. If $G$ is Class 2, then $G$ contains a critical subgraph $G^{*}$ with $\Delta\left(G^{*}\right)=\Delta(G)$.

The next lemma was proved in [5].

LEMMA 2.9. Let $G$ be a simple Class 2 graph with three vertices of maximum degree. Then $|V(G)|$ is odd and $G$ has three vertices of degree $|V(G)|-1$ and the rest have degree $|V(G)|-2$.

The next lemma is a generalization due to Berge[2] of a well-known theorem of Chvátal [10] on Hamiltonian circuits.

Lemмa $2 \cdot 10$. Let $G$ be a simple graph of order $n$ with degrees $d_{1} \leqslant d_{2} \leqslant \ldots \leqslant d_{n}$. Let $q$ be an integer with $0 \leqslant q \leqslant n-3$ and let $F$ be a subgraph of $G$ with $|E(F)|=q$ and such that the components of $F$ are paths. If, for $0<k<\frac{1}{2}(n-q)$,

$$
d_{k} \leqslant k+q \Rightarrow d_{n-k-q} \geqslant n-k,
$$

then $G$ contains a Hamiltonian circuit which contains $F$.

\section{The equivalence of Theorem 1 and Theorem 2}

It will be of great use in our proof of Theorem 1 if we know that Theorems 1 and 2 are equivalent to each other. In this section we prove this assertion.

LEMma 3.1. Let $G$ be a star multigraph with three vertices of degree $|V(G)|$, where $|V(G)|$ is odd and $\geqslant 5$, one vertex of degree $|V(G)|-2$, and the remainder of degree $|V(G)|-1$. Then $G=K_{2 n+1}^{*}$.

Proof. Let $v^{\prime}$ be the vertex of degree $|V(G)|-2$. Any vertex non-adjacent to $v^{*}$ has degree at most $|V(G)|-2$. Therefore each vertex, except possibly $v^{\prime}$, is adjacent to $v^{*}$. The three vertices of degree $|V(G)|$ each have at least one multiple edge on them, and so $v^{*}$ has maximum degree and is joined to the other two vertices, say $v_{1}$ and $v_{2}$, of maximum degree by multiple edges. Since $d\left(v^{*}\right) \leqslant|V(G)|, v^{*}$ is in fact joined to $v_{1}$ and $v_{2}$ by double edges, is not joined to $v^{\prime}$, and is joined to all other vertices by single edges, and $d\left(v^{*}\right)=|V(G)|$. A vertex $v \notin\left\{v^{*}, v_{1}, v_{2}\right\}$ is joined to all other vertices. Finally $v_{1}$ and $v_{2}$ are the two other vertices of degree $|V(G)|$ and are each joined to all other vertices. Therefore $G=K_{2 n+1}^{*}$.

LEMMA 3·2. Let $G$ be a star multigraph with star-centre $v^{*}$ and with at most three vertices of maximum degree, including $v^{*}$. If $G$ is just overfull, then $G$ satisfies (2iii).

Proof. Since $G$ is just overfull, $|\nabla(G)|$ is odd, say $|V(G)|=2 n+1$. If $|V(G)|=3$ then 
clearly $G$ satisfies (2iii), so suppose $|V(G)|>3$. If $G$ has at most two vertices of maximum degree, then, by Lemma $2 \cdot 3, G=K_{2 n+1}^{\dagger}$, so again $G$ satisfies (2iii). So now suppose that $G$ has three vertices of maximum degree.

Let the deficiency, $\operatorname{def}(G)$, of $G$ be defined by

$$
\operatorname{def}(G)=\sum_{\substack{v \in V(G) \\ d(v)<\Delta(G)-1}}(\Delta(G)-1-d(v))
$$

Then

$$
\begin{aligned}
n \Delta(G)+1 & =|E(G)| \\
& =\frac{1}{2}(3 \Delta(G)+(2 n-2)(\Delta(G)-1)-\operatorname{def}(G)) .
\end{aligned}
$$

Therefore

$$
2 n \Delta(G)+2=(2 n+1) \Delta(G)-2 n+2-\operatorname{def}(G)
$$

and so $\operatorname{def}(G)=\Delta(G)-2 n$. Therefore

$$
\Delta(G) \geqslant 2 n \text {. }
$$

Since $G$ is a star-multigraph, there are at most $\left(\begin{array}{c}2 n \\ 2\end{array}\right)$ edges between vertices of $V(G) \backslash\left\{v^{*}\right\}$. Therefore

Therefore

$$
n \Delta(G)+1 \leqslant|E(G)| \leqslant\left(\begin{array}{c}
2 n \\
2
\end{array}\right)+\Delta(G)
$$

so that

$$
n \Delta(G) \leqslant 2 n^{2}-n-1+\Delta(G)
$$$$
\Delta(G) \leqslant 2 n+1 \text {. }
$$

In view of (1), it now follows that either $G$ has three vertices of degree $|V(G)|$, one of degree $|V(G)|-2$, and the rest have degree $|V(G)|-1$, in which case, by Lemma 3.1, $G=K_{2 n+1}^{*}$, so that (2iii $c$ ) is satisfied, or $G$ has three vertices of degree $|V(G)|-1$ and the remainder have degree $|V(G)|-2$, so that ( 2 iii $d$ ) is satisfied.

This proves Lemma $3 \cdot 2$.

For use in the induction argument in Section 5, we need a more detailed assertion than simply that Theorems 1 and 2 are equivalent to each other. The assertion we need is the following lemma.

LEMMA 3.3. Let $1 \leqslant q \leqslant p$. Then Theorem 1 is true for $|V(G)|=p$ and $\Delta(G) \leqslant q$, or for $|V(G)|<p$, if and only if Theorem 2 is true when $|V(G)|=p$ and $\Delta(G) \leqslant q$ or when $|V(G)|<p$.

Proof. Both assertions are easily seen to be true if $0 \leqslant \Delta(G) \leqslant 2$. Suppose therefore that $\Delta(G) \geqslant 3$.

Throughout we shall assume that all graphs considered satisfy $|V(G)|=p$ and $\Delta(G) \leqslant q$, or $|V(G)|<p$.

(I) Suppose first that Theorem 1 is true when $|V(G)|=p$ and $\Delta(G) \leqslant q$, or when $V(G) \mid<p$. Then we wish to show Theorem 2 is true when $|V(G)|=p$ and $\Delta(G) \leqslant q$, or when $|V(G)|<p$.

If $(2 \mathrm{i})$ is true, so that $G$ is critical, then, from Theorem 1, it is clear that ( 2 iii) follows. If ( $2 \mathrm{iii})$ is true, then it is easy to see that both ( $2 \mathrm{i}$ ) and (2ii) are true. Finally, if (2ii) is true, then it follows from Lemma $3 \cdot 2$ that ( 2 iii) is true.

(II) Now suppose that Theorem 2 is true when $|V(G)|=p$ and $\Delta(G) \leqslant q$, or when $|V(G)|<p$. Then we wish to show that Theorem 1 is true when $|\nabla(G)|=p$ and $\Delta(G) \leqslant q$, or when $|V(G)|<p$. 
Suppose that $G$ satisfies the conditions of Theorem 1. If $G$ satisfies (1i), (1ii) or (1 iii) then it is easy to see that $G$ is Class 2. Now suppose that $G$ is Class 2. Then we shall show (using Theorem 2) that $G$ satisfies (1i), (1ii) or (1 iii). Since $G$ is Class 2, by Lemma 2.8, $G$ contains a critical subgraph $G^{*}$ with $\Delta\left(G^{*}\right)=\Delta(G)$. In Theorem 1, we are assuming that $G$ contains no subgraph on three vertices with $\Delta(G)+1$ vertices. Therefore, by Theorem $2,\left|V\left(G^{*}\right)\right|$ is odd $\left(=2 m+1\right.$, say) and either $\Delta\left(G^{*}\right)=2 m+1$ and $G=K_{2 m+1}^{\dagger}$ for some $m \geqslant 1$, or $\Delta\left(G^{*}\right)=2 m+1$ and $G=K_{2 m+1}^{*}$ for some $m \geqslant 2$, or $G^{*}$ has three vertices of degree $2 m$ and the remainder have degree $2 m-1$.

In the first case, since we are assuming in Theorem 1 that $G$ has exactly three vertices of maximum degree, it follows that $\left|V\left(G^{*}\right)\right|<|V(G)|$. Let $G_{2}$ be the subgraph of $G$ induced by $V(G) \backslash V\left(G^{*}\right)$. Then $G^{*}$ and $G_{2}$ are connected by a bridge $e$; thus $G$ satisfies (1 iii) (with $G_{1}=G^{*}$ ).

In the second case, either $\left|V\left(G^{*}\right)\right|=|V(G)|$, in which case $G$ satisfies (1i) or $\left|V\left(G^{*}\right)\right|<|V(G)|$. Let $G_{2}$ be the subgraph of $G$ induced by $V(G) \backslash V\left(G^{*}\right)$. Then, since $G$ is connected and has exactly three vertices of maximum degree, $G^{*}$ is joined to $G_{2}$ by a bridge $e$ (which is incident with the vertex of degree $2 m-1$ in $G$ ); thus $G$ again satisfies ( 1 iii) (with $G_{1}=G^{*}$ ).

In the third case, since $G$ is connected and has exactly three vertices of maximum degree, $V(G)=V\left(G^{*}\right)$, so $G=G^{*}$. Then $G$ satisfies (1ii).

This proves Lemma 3.3.

\section{A bound on the maximum degree}

For a critical star multigraph $G$, when there are only three vertices of maximum degree, including $v^{*}$, the bound on $\Delta(G)$ in Lemma $2 \cdot 4$ reduces to

$$
\Delta(G) \leqslant|V(G)|+\mu-2,
$$

where $\mu$ is the maximum multiplicity. Our task in this section is to reduce this bound.

LEMMa 4-1. Let $G$ be a critical star multigraph with star-centre $v^{*}$ and with three vertices, $v_{1}, v_{2}, v^{*}$, of maximum degree. Then either (i) $\Delta(G) \leqslant|V(G)|-1$ and $d(v) \geqslant \Delta(G)-1$ $(\forall v \in V(G))$ (so that $|V(G)|$ is odd), or (ii) $G=K_{2 n+1}^{*}$ for some $n \geqslant 2$.

Proof. By Lemma 2.9, if $G$ is a simple graph, then $|V(G)|$ is odd and $G$ has three vertices of degree $|V(G)|-1$ and the rest have degree $|V(G)|-2$. Thus (i) is satisfied. Therefore we may suppose that $|V(G)|$ is not simple. Then it follows from Lemma 2.7 that $G$ has a vertex $v_{0}$ which is non-adjacent to $v^{*}$; we note that then

$$
d\left(v_{0}\right) \leqslant|V(G)|-2 .
$$

If $d(v) \geqslant \Delta(G)-1(\forall v \in V(G))$, then it follows that $\Delta(G)-1 \leqslant d\left(v_{0}\right) \leqslant|V(G)|-2$, so $\Delta(G) \leqslant|V(G)|-1$. Thus Case (i) of the lemma follows.

Suppose from now that there is a vertex $u \in V(G)$ such that $d(u)=\Delta(G)-x$, for some $x \geqslant 2$. Let $u$ be joined to $v^{*}$ by $m^{*}$ edges. As $G$ is critical, $u$ cannot be joined solely to $v^{*}$, so let $u$ also be joined to vertices $w_{1}, \ldots, w_{t}$, where $t \geqslant 1$. By Lemma $2 \cdot 5$,

$$
d^{*}\left(w_{i}\right) \geqslant \Delta(G)-d(u)+1=x+1
$$

for $1 \leqslant i \leqslant t$. Therefore, for $1 \leqslant i \leqslant t$,

$$
m\left(v^{*}, w_{i}\right) \geqslant\left\{\begin{array}{lll}
x-1 & \text { if } & w_{i} \notin\left\{v_{1}, v_{2}\right\} \\
x & \text { if } & w_{i} \in\left\{v_{1}, v_{2}\right\}
\end{array}\right.
$$


By Lemma $2 \cdot 5$, again, $d^{*}(v) \geqslant 2$ for all $v \in V(G) \backslash\left\{v^{*}\right\}$. But since $d^{*}\left(v_{1}\right) \geqslant 2, d^{*}\left(v_{2}\right) \geqslant 2$ and $m\left(v_{1}, v_{2}\right) \leqslant 1$, it follows that $d^{*}\left(v^{*}\right) \geqslant 2$ also. Thus

$$
d^{*}(v) \geqslant 2 \quad(\forall v \in V(G)) .
$$

Whatever the value of $\left|\left\{v_{1}, v_{2}\right\} \cap\left\{w_{1}, \ldots, w_{t}\right\}\right|$, we have

$$
\Delta(G)=d^{*}(v) \geqslant m^{*}+t(x-1)+2 .
$$

Since $d(u)=\Delta(G)-x=m^{*}+t$, it follows that $m^{*}=\Delta(G)-x-t$, and so, after substituting, we find that $2(t-1) \geqslant x(t-1)$. Therefore either $t=1$ or $x=2$.

Suppose that $t=1$. Consider the possibility that $w_{1} \in\left\{v_{1}, v_{2}\right\}$. Then we may assume that $w_{1}=v_{1}$. By Lemma $2 \cdot 5, d^{*}\left(w_{1}\right) \geqslant x+1$, and so it follows that $m\left(v^{*}, w_{1}\right) \geqslant x$. Also by Lemma $2 \cdot 5, d^{*}\left(v_{2}\right) \geqslant 2$, and so $m\left(v^{*}, v_{2}\right) \geqslant 1$. Since $d(u)=\Delta(G)-x$, we have $m\left(v^{*}, u\right)=\Delta(G)-x-1$. Since there are exactly $\Delta(G)$ edges incident with $v^{*}$, it follows that $m\left(v^{*}, w_{1}\right)=x, m\left(v^{*}, v_{2}\right)=1$ and that $v^{*}$ is joined to no vertex other than $u, w_{1}$ and $v_{2}$. Consequently $m\left(v_{1}, v_{2}\right)=1$. Thus, within the subgraph induced by $\left\{v^{*}, w_{1}, v_{2}, u\right\}$, there are $x+2 \geqslant 4$ edges incident with $w_{1}$ and 2 edges incident with $v_{2}$. But any further vertex satisfies $d^{*}(v) \geqslant 2$, is not joined to $v^{*}$, and so is joined to $v_{1}$ and $v_{2}$. Therefore $d\left(w_{1}\right) \geqslant d\left(v_{2}\right)+2$, so $d\left(w_{1}\right) \neq d\left(v_{2}\right)$, a contradiction.

Now suppose that $t=1$ but $w_{1} \notin\left\{v_{1}, v_{2}\right\}$. By Lemma 2.5, $d^{*}\left(w_{1}\right) \geqslant x+1, d^{*}(v) \geqslant 2$ and $d^{*}\left(v_{2}\right) \geqslant 2$, and so $m\left(v^{*}, w_{1}\right) \geqslant x-1, m\left(v^{*}, v_{1}\right) \geqslant 1$ and $m\left(v^{*}, v_{2}\right) \geqslant 1$. As above, $m\left(v^{*}, u\right)=\Delta(G)-x-1$. As there are exactly $\Delta(G)$ edges incident with $v^{*}$, it follows that $m\left(v^{*}, w_{1}\right)=x-1, m\left(v^{*}, v_{1}\right)=m\left(v^{*}, v_{2}\right)=1$, and that $v^{*}$ is joined to no other vertex. Consequently $m\left(v_{1}, v_{2}\right)=1$. Since $d^{*}(v) \geqslant 2$ for all $v \in V(G)$, it follows that any vertex $p \in V \backslash\left\{v^{*}, u, v_{1}, v_{2}\right\}$ is joined to both $v_{1}$ and $v_{2}$, and consequently

$$
\Delta(G)=d\left(v_{1}\right)=|V(G)|-2 .
$$

But by Lemma $2 \cdot 5, d(p) \geqslant \Delta(G)-d^{*}\left(v_{1}\right)+1=\Delta(G)-1=|V(G)|-3$. Thus $p$ is joined to all vertices except $v^{*}$ and $u$. Therefore

$$
d\left(w_{1}\right)=(|V(G)|-5)+3+(x-1) \leqslant \Delta(G)-1=|V(G)|-3 .
$$

Therefore $x=0$. But $x \geqslant 2$, a contradiction. Thus $t \neq 1$.

Now suppose that $t \geqslant 2$ and $x=2$. Then $d(u)=\Delta(G)-2$. Suppose first that $\left\{v_{1}, v_{2}\right\} \subseteq\left\{w_{1}, \ldots, w_{t}\right\}$. We may assume that $w_{1}=v_{1}$ and $w_{2}=v_{2}$. Since

$$
d^{*}\left(w_{i}\right) \geqslant x+1 \geqslant 3 \quad(1 \leqslant i \leqslant t),
$$

it follows that $m\left(v^{*}, w_{i}\right) \geqslant 1 \quad(3 \leqslant i \leqslant t), m\left(v^{*}, v_{1}\right) \geqslant 2$ and $m\left(v^{*}, v_{2}\right) \geqslant 2$. Since $m\left(v^{*}, u\right)=\Delta(G)-x-t=\Delta(G)-2-t$ and $d\left(v^{*}\right)=\Delta(G)$, it follows that $m\left(v^{*}, w_{i}\right)=1$ $(3 \leqslant i \leqslant t)$, that $m\left(v^{*}, v_{1}\right)=m\left(v^{*}, v_{2}\right)=2$ and that $v^{*}$ is joined to no other vertices. Therefore, as before, $v_{1}$ is joined to all vertices $v \in V(G) \backslash\left\{v^{*}, u, v_{1}, v_{2}, w_{3}, \ldots, w_{t}\right\}$. Since $d^{*}\left(v_{1}\right)=3$ and $m\left(v^{*}, v_{1}\right)=2$, it follows that $m\left(v_{1}, v_{2}\right)=1$. Since, for $3 \leqslant i \leqslant t, d^{*}\left(w_{i}\right) \geqslant 3$ and $m\left(v^{*}, w_{i}\right)=1$, it follows that $m\left(v_{1}, w_{i}\right)=m\left(v_{2}, w_{i}\right)=1$ and $d^{*}\left(w_{i}\right)=3$. Therefore $\Delta(G)=d\left(v_{1}\right)=|V(G)|$. Now consider the vertex $v_{0}$, which is non-adjacent to $v^{*}$. Then $v_{0} \notin\left\{v_{1}, v_{2}, v^{*}, w_{3}, \ldots, w_{t}\right\}$. If $v_{0} \neq u$, then $v_{0}$ is non-adjacent to both $v^{*}$ and $u_{0}$, so $d\left(v_{0}\right) \leqslant|V(G)|-3$. However, as $v_{0}$ is adjacent to $v_{1}$ and $d^{*}\left(v_{1}\right)=3$, it follows from Lemma $2 \cdot 5$ that $d\left(v_{0}\right) \geqslant \Delta(G)-2=|V(G)|-2$, a contradiction. Therefore $v_{0}=u$. Then $m^{*}=\Delta(G)-2-t=0$, so $t=\Delta(G)-2=|V(G)|-2$, so $v^{*}$ is joined to every vertex except $v_{0}$, and is joined to $v_{1}$ and $v_{2}$ by two edges, and to every other vertex by one 
edge. Since $d(u)=|V(G)|-2, v_{0}=u$ is joined to every vertex except $v^{*}$. For $3 \leqslant i \leqslant t$, $w_{i}$ is adjacent to $u$, so by Lemma $2 \cdot 5, d\left(w_{i}\right) \geqslant \Delta(G)-d^{*}(u)+1=|V(G)|-1$, so $w_{i}$ is joined to every vertex. Therefore $G=K_{m}^{*}$ for some $m \geqslant 4$.

We now show that $m$ is not even. Suppose, to the contrary, that $m=2 p$. If $p=2$ then $K_{2 p}^{*}$ is Class 2 , but is not critical, a contradiction. Now suppose that $p \geqslant 3$. Then we obtain a contradiction by showing that $K_{2 p}^{*}$ is Class 1 . Let $K_{2 p}$ be edge-coloured with colours $c_{1}, \ldots, c_{2 p-1}$, and let $v^{*}, v_{0}, v_{1}, v_{2}, v^{\prime}$ be distinct vertices such that $v^{*} v_{0}$ and $v_{1} v^{\prime}$ are both coloured $c_{2 p-1}$. Then recolour $v_{1} v^{\prime}$ with $c_{2 p}$, remove the edge $v^{*} v_{0}$, insert an extra edge joining $v^{*}$ to $v_{1}$ coloured $c_{2 p-1}$, and an extra edge joining $v^{*}$ to $v_{2}$ coloured $c_{2 p}$. Then $K_{2 p}^{*}$ is edge-coloured with $2 p$ colours, and so $K_{2 p}^{*}$ is Class 1 . Therefore $G \neq K_{2 p}^{*}$. Therefore $G=K_{2 n+1}^{*}$ for some $n \geqslant 2$, so $G$ satisfies Case (ii) of this lemma.

Now suppose that $\left|\left\{v_{1}, v_{2}\right\} \cap\left\{w_{1}, \ldots, w_{t}\right\}\right|=1$. We may suppose that $v_{1}=w_{1}$ and that $v_{2} \notin\left\{w_{1}, \ldots, w_{t}\right\}$. We have $m\left(v^{*}, u\right)=\Delta(G)-t-2$ and, by Lemma $2 \cdot 5$,

$$
d^{*}\left(w_{i}\right) \geqslant 3 \quad(1 \leqslant i \leqslant t),
$$

so $m\left(v^{*}, v_{1}\right) \geqslant 2$ and $m\left(v^{*}, w_{i}\right) \geqslant 1(2 \leqslant i \leqslant t)$. We also have $d^{*}\left(v_{2}\right) \geqslant 2$, so $m\left(v^{*}, v_{2}\right) \geqslant 1$. As $d\left(v^{*}\right)=\Delta(G)$, it follows that $m\left(v^{*}, v_{1}\right)=2, m\left(v^{*}, w_{i}\right)=1(2 \leqslant i \leqslant t), m\left(v^{*}, v_{2}\right)=1$, $m\left(v_{1}, v_{2}\right)=1, v^{*}$ is joined to no other vertex, and that each vertex not in

$$
\left\{v^{*}, u, v_{1}, v_{2}, w_{2}, \ldots, w_{t}\right\}
$$

is joined to $v_{1}$ and $v_{2}$. But then within the subgraph induced by $\left\{v^{*}, u, v_{1}, v_{2}, w_{2}, \ldots, w_{t}\right\}$, $v_{1}$ has $t+3$ edges on it and $v_{2}$ has $t+1$ edges on it, so $\Delta(G)=d\left(v_{1}\right) \neq d\left(v_{2}\right)=\Delta(G)$, a contradiction. Therefore $\left|\left\{v_{1}, v_{2}\right\} \cap\left\{w_{1}, \ldots, w_{t}\right\}\right| \neq 1$.

Finally, suppose that $\left\{v_{1}, v_{2}\right\} \cap\left\{w_{1}, \ldots, w_{t}\right\}=\varnothing$. As in earlier cases, it follows that $m\left(v^{*}, u\right)=\Delta(G)-t-2, \quad m\left(v^{*}, v_{1}\right) \geqslant 1, m\left(v^{*}, v_{2}\right) \geqslant 1$ and $m\left(v^{*}, w_{i}\right) \geqslant 1 \quad(1 \leqslant i \leqslant t)$. Therefore, as $d\left(v^{*}\right)=\Delta(G)$, it follows that

$$
m\left(v^{*}, u\right)=\Delta(G)-t-2, \quad m\left(v^{*}, v_{1}\right)=m\left(v^{*}, v_{2}\right)^{\prime}=m\left(v^{*}, w_{i}\right)=1 \quad(1 \leqslant i \leqslant t),
$$

$m\left(v_{1}, v_{2}\right)=1, v^{*}$ is joined to no other vertices, and all vertices of

$$
V(G) \backslash\left\{v^{*}, u, v_{1}, v_{2}, w_{1}, \ldots, w_{t}\right\}
$$

are joined to $v_{1}$ and $v_{2}$. Since $m\left(v_{1}, v^{*}\right)=1$ and $v_{1}$ is joined to all vertices except $u$, we have $\Delta(G)=d\left(v_{1}\right)=|V(G)|-2$. By Lemma $2 \cdot 5$, it follows that if

$$
v \in V(G) \backslash\left\{v_{1}, v_{2}, v^{*}, u\right\},
$$

then $d(v)=\Delta(G)-1=|V(G)|-3$. If $p \in V(G) \backslash\left\{v_{1}, v_{2}, v^{*}, u, w_{1}, \ldots, w_{t}\right\}$, then $p$ is joined to all vertices except $u$ and $v^{*}$. As the vertices $w_{1}, \ldots, w_{t}$ are each joined to each vertex of $V(G) \backslash\left\{w_{1}, \ldots, w_{t}\right\}$ by a single edge, $w_{1}, \ldots, w_{t}$ induce a graph which is regular of degree $t-3$. Therefore $t \geqslant 3$. We also have

$$
m\left(v^{*}, u\right)=\Delta(G)-t-2=|V(G)|-t-4 \leqslant|V(G)|-7 .
$$

Since $G$ is not a simple graph, it follows that $m\left(v^{*}, u\right) \geqslant 2$. Therefore $|V(G)| \geqslant 9$.

Suppose first that $|V(G)|$ is even, $|V(G)|=2 n$ say. Let $G^{\prime}$ be a graph obtained from $G$ by replacing all the edges between $u$ and $v^{*}$ by a single edge. Let $d_{1}^{\prime} \leqslant d_{2}^{\prime} \leqslant \ldots \leqslant d_{2 n}^{\prime}$ be the degrees of $G^{\prime}$. Then, as $n \geqslant 5$, we have

$$
d_{1}^{\prime} \geqslant 4, \quad d_{2}^{\prime} \geqslant 6, \quad d_{3}^{\prime} \geqslant 2 n-3, \ldots, d_{2 n-2}^{\prime} \geqslant 2 n-3, \quad d_{2 n-1}^{\prime}=d_{2 n}^{\prime}=2 n-2,
$$


and the condition

$$
d_{k}^{\prime} \leqslant k+1 \Rightarrow d_{2 n-k-1}^{\prime} \geqslant 2 n-k \quad(1 \leqslant k \leqslant n-1)
$$

is satisfied, so, by Lemma $2 \cdot 10, G^{\prime}$ has a Hamiltonian circuit which includes the edge $v_{1} v_{2}$, and hence $G$ has a 1-factor $F$ including $v_{1} v_{2}$. Now $G \backslash F$ has three vertices $v^{*}, v_{1}, v_{2}$ of maximum degree $\Delta(G)-1$ and $d_{G{ }_{F}}^{*}\left(v_{1}\right)=d_{G{ }_{F}}^{*}\left(v_{2}\right)=1$. By Lemma $2 \cdot 8$, if $G \backslash F$ were Class 2 , then $G \backslash F$ would contain a critical subgraph $H$ with $\Delta(H)=\Delta(G \backslash F)$. But by Lemma $2 \cdot 5, v_{1}, v_{2} \notin V(H)$, and so $H$ has just one vertex, namely $v^{*}$, of maximum degree. Since $\Delta(G)=|V(G)|-2 \geqslant 8>2, H$ contains no subgraph on three vertices with $\Delta(H)+1$ edges [the only possibility for this would include $v^{*}$ as one of the three vertices, but the neighbours of $v^{*}$ are mutually non-adjacent]. Therefore, by Lemma $2 \cdot 3$, $H$ is Class 1, a contradiction. Therefore $|V(G)|$ is not even.

Next suppose that $|V(G)|$ is odd, $|V(G)|=2 n+1$, say. Let $G^{\prime}$ be a graph obtained from $G$ by replacing all the edges between $u$ and $v^{*}$ by a single edge. Let $G^{\prime \prime}$ be obtained from $G^{\prime}$ by adjoining the further edge $v_{2} u$. Let $d_{1}^{\prime \prime} \leqslant d_{2}^{\prime \prime} \leqslant \ldots \leqslant d_{2 n-1}^{\prime \prime}$ be the degree sequence of $G^{\prime \prime}$. Then, as $n \geqslant 4$, we have

$$
d_{1}^{\prime \prime} \geqslant 5, \quad d_{2}^{\prime \prime} \geqslant 6, \quad d_{3}^{\prime \prime} \geqslant 2 n-2, \ldots, d_{2 n-1}^{\prime \prime} \geqslant 2 n-2, \quad d_{2 n}^{\prime \prime}=2 n-1, \quad d_{2 n+1}^{\prime \prime}=2 n,
$$

and $G^{\prime \prime}$ satisfies the condition

$$
d_{k}^{\prime \prime} \geqslant k+2 \Rightarrow d_{2 n-1-k}^{n} \geqslant 2 n+1-k \quad(1 \leqslant k \leqslant n-1) .
$$

Therefore, by Lemma $2 \cdot 10, G^{\prime \prime}$ has a Hamiltonian circuit which includes the path $v_{1} v_{2}, v_{2} u$. Therefore $G$ has a near 1 -factor $F$ which includes the edge $v_{1} v_{2}$ and does not contain an edge incident with $u$. Then $G \backslash F$ has three vertices $v^{*}, v_{1}, v_{2}$ of maximum degree $\Delta(G)-1$ and $d_{G \backslash F}^{*}\left(v_{1}\right)=d_{G \backslash F}^{*}\left(v_{2}\right)=1$. We now obtain a contradiction as above.

\section{Proof of Theorems 1 and 2}

By Lemma 3·3, Theorems 1 and 2 are equivalent. Formally we shall prove Theorem 2, but we shall make use of this equivalence. To see that ( $2 \mathrm{ii}) \Rightarrow$ (2 iii) we use Lemma $3 \cdot 2$. It is easy to see that ( $2 \mathrm{iii}) \Rightarrow(2 \mathrm{ii})$ and that (2ii) $\Rightarrow(2 \mathrm{i})$. What we have to prove is that (2i) $\Rightarrow$ (2 ii).

Let $G$ be a critical star multigraph with star centre $v^{*}$ with at most three vertices of maximum degree, including $v^{*}$. For simple graphs, the truth of Theorem 2 follows from Lemma $2 \cdot 9$. Therefore we shall assume that $G$ has at least one multiple edge. If $|V(G)|=3$, then, since $G$ is a star multigraph, $|E(G)|=\Delta(G)+1$. Suppose from now on that $|V(G)|>3$. Then $G$ contains no subgraph on three vertices with $\Delta(G)+1$ edges, for otherwise $G$ would not be critical. It therefore follows from Lemma $2 \cdot 3$ that Theorem 2 is true when $G$ has at most two vertices of maximum degree. Therefore we shall also assume that $G$ has three vertices of maximum degree, $v^{*}, v_{1}$ and $v_{2}$.

It follows from Lemma $4 \cdot 1$ that either $G$ is $K_{2 n+1}^{*}$ for some $n \geqslant 2$, in which case $G$ is clearly overfull, or $G$ has three vertices of degree $\Delta(G)$ and the rest have degree $\Delta(G)-1$, where $\Delta(G) \leqslant|V(G)|-1$. In this case, since $|E(G)|=\frac{1}{2}(|V(G)|(\Delta(G)-1)+3)$, it follows that $|V(G)|$ is odd and $\Delta(G)$ is even. If $\Delta(G)=|V(G)|-1$ then $G$ is just overfull, as required. We suppose from now that $\Delta(G) \leqslant|V(G)|-2$. Then, in fact, we have

$$
\Delta(G) \leqslant|V(G)|-3
$$


By Lemma 2.2, we may assume that

$$
\left[\frac{2}{3}|V(G)|\right] \leqslant \Delta(G)
$$

It follows from (2) and (3) that $|V(G)| \geqslant 9$, and that if $|V(G)|=9$ then $\Delta(G)=6$.

The proof proceeds by induction. We asssume that Theorem 2 (and therefore, by Lemma 3.3, Theorem 1 also) is true for lower values of $|V(G)|$, or for the given value of $|V(G)|$ and lower values of $\Delta(G)$.

As in Lemma $4 \cdot 1$

$$
d^{*}(v) \geqslant 2 \quad(\forall v \in V(G)) .
$$

Therefore, as there are three vertices of maximum degree,

$$
3 \Delta(G)=\sum_{v \in V(G)} d^{*}(v) \geqslant 2|V(G)| .
$$

Let the excess $\epsilon(G)$ of $G$ be defined by

$$
\epsilon(G)=3 \Delta(G)-2|V(G)| .
$$

Thus $\epsilon(G)$ measures the amount by which $\sum_{v_{\epsilon} V(G)} d^{*}(v)$ exceeds $2|V(G)|$. By (2) we have $\epsilon(G) \leqslant \Delta(G)-2(|V(G)|-\Delta(G)) \leqslant \Delta(G)-6$, so

$$
0 \leqslant \epsilon(G) \leqslant \Delta(G)-6
$$

with $\epsilon(G)=\Delta(G)-6$ only if $\Delta(G)=|V(G)|-3$. Therefore

$$
\sum_{\substack{w \in V(G) \backslash v^{*} \\ m\left(v^{*}, w\right) \geq 2}}\left(m_{G}\left(v^{*}, w\right)-2\right) \leqslant \Delta(G)-6,
$$

with equality only if $\Delta(G)=|V(G)|-3$.

There is a vertex $a$ which is non-adjacent to $v^{*}$. Since $d_{G}(a)=\Delta(G)-1 \leqslant|V(G)|-4$, there is a vertex $b \notin\left\{v_{1}, v_{2}, v^{*}\right\}$ which is non-adjacent to $a$. Our first move is to show that there is a near 1-factor $F$ which does not include an edge which is incident with $b$, but which does include the edge $a v_{1}$.

From $G$, form a graph $G^{+}$by inserting an edge $a b$. From $G^{+}$form a graph $G^{\prime}$ by replacing each multiple edge on $v^{*}$ by a single edge. Let the vertices of $G^{\prime}$ be

$$
w_{1}, w_{2}, \ldots, w_{2 n+1}
$$

and have degrees $d_{1}^{\prime} \leqslant d_{2}^{\prime} \leqslant \ldots \leqslant d_{2 n+1}^{\prime}$. We may suppose that $w_{1}=v^{*}$ except perhaps when there is only one multiple edge, between $v^{*}$ and, say, $w_{1}$, where $d_{G}\left(w_{1}\right)=\Delta(G)-1$, and then we may take $v^{*}$ to be $w_{2}$.

If $d^{\prime}\left(v^{*}\right) \leqslant 3$ then, since $d^{*}\left(v_{1}\right) \geqslant 2$ and $d^{*}\left(v_{2}\right) \geqslant 2$, it follows that $\epsilon(G) \geqslant \Delta(G)-6$. From (4) it then follows that $\epsilon(G)=\Delta(G)-6$ and that

and

$$
d^{\prime}\left(v^{*}\right)=3, \quad m_{G}\left(v^{*}, v_{1}\right)=m_{G}\left(v^{*}, v_{2}\right)=2, \quad m_{G}\left(v^{*}, w_{2}\right)=\Delta(G)-4,
$$

$$
\Delta(G)=|V(G)|-3 .
$$

But if $v \in V(G) \backslash\left\{v^{*}, w_{2}, v_{1}, v_{2}\right\}$, then $v$ is joined to $v_{1}$, so $d_{G}\left(v_{1}\right)=|V(G)|-2$, a contradiction. Therefore

$$
d^{\prime}\left(v^{*}\right) \geqslant 4 \text {. }
$$


For $1 \leqslant i \leqslant 2 n+1$, if $w_{i} \neq v^{*}$, let $\mu_{i}$ be the number of edges in $G^{+}$joining $w_{i}$ to $v^{*}$. By Lemma 2.10, if

$$
d_{k}^{\prime} \leqslant k+2 \Rightarrow d_{2 n-1-k}^{\prime} \geqslant 2 n+1-k \quad(1 \leqslant k \leqslant n-1),
$$

then $G^{\prime}$ contains a Hamiltonian circuit including the path $v_{1} a, a b$, and so $G$ contains a near 1 -factor $F$ including the edge $a v_{1}$ but not including any edge incident with $b$.

First suppose, for some $k$ with $3 \leqslant k \leqslant n-1$, that $d_{k}^{\prime} \leqslant k+2$; we shall consider later the possibilities when $k=1$ or 2 .

Suppose that

$$
d_{G}\left(v^{*}\right)=\sum_{\substack{i=1 \\ w_{s} \neq v^{*}}}^{k} \mu_{i}
$$

Then $v_{1}, v_{2} \in\left\{w_{1}, \ldots, w_{k}\right\}$. Since $d_{k}^{\prime} \leqslant k+2$ and $d_{k}^{\prime}=d_{G}\left(w_{k}\right)-\mu_{k}+1 \geqslant \Delta(G)-\mu_{k}$, it follows that $\mu_{k} \geqslant \Delta(G)-k-2$. Bearing in mind that $d_{G}\left(v_{1}\right)=d_{G}\left(v_{2}\right)=\Delta(G)$ and that $d_{1}^{\prime} \leqslant \ldots \leqslant d_{k}^{\prime}$, we have

It follows that

$$
\Delta(G)-\mu_{i} \leqslant \begin{cases}k+2 & \text { if } \mu_{i} \notin\left\{v^{*}, v_{1}, v_{2}\right\} \text { and } 1 \leqslant i \leqslant k, \\ k+1 & \text { if } \mu_{i}=v_{1} \text { or } v_{2} .\end{cases}
$$

and so, for $k \geqslant 3$,

$$
(k-1) \Delta(G)-\Delta(G)=\sum_{\substack{i=1 \\ w_{i} \neq v^{*}}}^{k}\left(\Delta(G)-\mu_{i}\right) \leqslant(k-1)(k+2)-2
$$

$$
\Delta(G) \leqslant k+3+\left[\frac{2}{k-2}\right] .
$$

Using (3), since $k \leqslant n-1$, we have

$$
\frac{4 n+2}{3} \leqslant\left\lceil\frac{2}{3}|V(G)|\right\rceil \leqslant \Delta(G) \leqslant k+3+\left\lfloor\frac{2}{k-2}\right\rfloor \leqslant n+2+\left\lfloor\frac{2}{k-2}\right\rfloor .
$$

Now suppose that

$$
d_{G}\left(v^{*}\right)=\left(\sum_{\substack{i=1 \\ w_{i} \neq v^{*}}}^{k} \mu_{i}\right)+1
$$

Then $\left\{v_{1}, v_{2}\right\} \cap\left\{w_{1}, \ldots, w_{k}\right\} \neq \varnothing$. In a similar way, we obtain

from which (8) again follows.

$$
(k-1) \Delta(G)-(\Delta(G)-1)=\sum_{\substack{i=1 \\ w_{i} \neq v^{*}}}^{k}\left(\Delta(G)-\mu_{i}\right) \leqslant(k-1)(k+2)-1,
$$

Lastly, if

$$
d_{a}\left(v^{*}\right) \leqslant\left(\sum_{\substack{i=1 \\ w_{i} \neq v^{*}}}^{k} \mu_{i}\right)+2
$$

then we obtain similarly

and so (8) follows again.

$$
(k-1) \Delta(G)-(\Delta(G)-2) \leqslant \sum_{\substack{i=1 \\ u_{i} \neq v^{*}}}^{k}\left(\Delta(G)-\mu_{i}\right) \leqslant(k-1)(k+2),
$$

If $k \geqslant 5$, then it follows from (8) that $n \leqslant 4$, so $k \leqslant n-1 \leqslant 3$, a contradiction. Therefore $k=3$ or 4 . From (8) it now follows that $\Delta(G) \leqslant 8$. Applying (8) yet again, 
$(4 n+2) / 3 \leqslant 8$, and so $n \leqslant 5$. By (3) and (8), it follows that the values of $(k, n, \Delta(G))$ we must consider are $(4,5,8),(3,4,6)$ and $(3,5,8)$. We consider these cases separately.

First consider the case when $(k, n, \Delta(G))=(4,5,8)$. Then $d_{1}^{\prime} \leqslant d_{2}^{\prime} \leqslant d_{3}^{\prime} \leqslant d_{4}^{\prime} \leqslant 6$ and $w_{1}=v^{*}$. Bearing in mind that $m_{G}\left(v^{*}, v_{1}\right) \geqslant 1$ and $m_{G}\left(v^{*}, v_{2}\right) \geqslant 1$, the only possibilities are either that $(a) d^{\prime}\left(v^{*}\right)=5$ and $v^{*}$ has on it two edges of multiplicity 1 and three edges of multiplicity 2 , or that $(b) d^{\prime}\left(v^{*}\right)=4$ and there is one edge of multiplicity 1 , two edges of multiplicity 2 and one edge of multiplicity 3 on $v^{*}$, or that $(c) d^{\prime}\left(v^{*}\right)=3$ and there is one edge of multiplicity 2 and two edges of multiplicity 3 on $v^{*}$. In case $(a)$, we have $d_{1}^{\prime}=5, d_{2}^{\prime}=d_{3}^{\prime}=d_{4}^{\prime}=6, d_{5}^{\prime}=7$, and so $G^{+}$satisfies the Chvátal-Berge condition (7). In case $(b)$, we have $d_{1}^{\prime}=4, d_{2}^{\prime}=d_{3}^{\prime}=d_{4}^{\prime}=6, d_{5}^{\prime}=7$ and again (7) is satisfied. Finally, case $(c)$ does not in fact arise, because $\epsilon(G)=3 \Delta(G)-2|V(G)|=2$, so that $d^{*}(v)=2$ for all except at most two vertices $v$.

Next consider the case when $(k, n, \Delta(G))=(3,5,8)$. Then $d_{1}^{\prime} \leqslant d_{2}^{\prime} \leqslant d_{3}^{\prime} \leqslant 5$ and $w_{1}=v^{*}$. Bearing in mind that $m_{G}\left(v^{*}, v_{1}\right) \geqslant 1$ and $m_{G}\left(v^{*}, v_{2}\right) \geqslant 1$, and that, by $(6)$, $d^{\prime}\left(v^{*}\right) \geqslant 4$, the only possibility is that $d\left(v^{*}\right)=4$ and $v^{*}$ has two edges of multiplicity 1 and two edges of multiplicity 3 on it. Let $d_{1}^{\prime \prime} \leqslant \ldots \leqslant d_{10}^{\prime \prime}$ be the degrees of $G^{\prime} \backslash\left\{v_{1}\right\}$. Since, as above, $d^{*}(v)=2$ for all except at most two vertices $v, w_{2}$ and $w_{3}$ cannot be joined to either $v_{1}$ or $v_{2}$. Therefore $d_{1}^{\prime \prime}=3, d_{2}^{\prime \prime}=5, d_{3}^{\prime \prime}=5$ and $d_{4}^{\prime \prime}=6$. Therefore the condition

$$
d_{k}^{\prime \prime} \leqslant k+1 \Rightarrow d_{2 n-1-k}^{\prime \prime} \geqslant 2 n-k \quad(1 \leqslant k \leqslant n-1)
$$

is satisfied with $n=5$, and so, by Lemma $2 \cdot 10, G^{\prime} \backslash\left\{v_{1}\right\}$ has a Hamiltonian circuit containing the edge $a b$. Therefore $G^{\prime} \backslash\left\{v_{1}\right\}$ has a 1 -factor containing $a b$. But $a$ is adjacent to $v_{1}$ in $G^{+}$, so $G^{+}$(and therefore $G$ ) has a near 1 -factor $F$ containing the edge $a v_{1}$ and avoiding the vertex $b$.

Lastly consider the case when $(k, n, \Delta(G))=(3,4,6)$. In this case we obtain a contradiction by arguing directly that $G$ is Class 1 . Since $\epsilon(G)=3 \Delta(G)-2|V(G)|=0$ and $d^{*}(v) \geqslant 2(\forall v \in V(G))$, it follows that $d_{G}\left(v^{*}\right)=2(\forall v \in V(G))$. Therefore there is exactly one edge between any two of $v^{*}, v_{1}, v_{2}$, there are no edges of multiplicity greater than 2 and any vertex joined to $v^{*}$ by a double edge is not joined to $v_{1}$ or $v_{2}$. Either $(a) v^{*}$ has four edges of multiplicity 1 and one edge of multiplicity 2 , or $(b) v^{*}$ has two edges of multiplicity 1 and two edges of multiplicity 2 . In case $(a) G \mid v^{*}$ has degrees $3,4,4$, $5,5,5,5,5$. By Lemma $2 \cdot 10, G \backslash v^{*}$ has a Hamiltonian circuit containing the edge $v_{1} v_{2}$. Consequently $G$ has a near 1 -factor $F^{\prime}$ containing one edge from the double edge on $v^{*}$ and the edge $v_{1} v_{2}$. But $G \backslash F^{\prime}$ is a simple graph of order 9 and maximum degree 5 and is not overfull. Therefore, by Lemma $2 \cdot 1, G \backslash F$ (and therefore $G$ ) is Class 1 , a contradiction. In case $(b), G \backslash v^{*}$ has degree sequence $(3,3,5,5,5,5,5,5)$. Let $v_{7}$ and $v_{8}$ be the vertices joined to $v^{*}$ by a double edge. By Lemma $2 \cdot 10, G \backslash v^{*}$ has a Hamiltonian circuit. Therefore $G$ has a 1 -factor $F_{1}$ containing one of the edges joining $v^{*}$ to $v_{8}$, an edge incident with $v_{1}$ and an edge incident with $v_{2}$. Then $G \backslash F_{1}$ has four vertices $v^{*}, v_{1}, v_{2}$, and, say, $w$, of degree 5 , and the remaining vertices have degree 4 . If $w=v_{7}$, then the degree sequence of $\left(G \backslash F_{1}\right) \backslash v^{*}$ is $(3,3,4,4,4,4,4,4)$. By Lemma $2 \cdot 10$, this has a Hamiltonian circuit, and so $G$ has a near 1 -factor $F_{2}$ containing an edge joining $v^{*}$ to $v_{7}$, an edge incident with $v_{1}$, and an edge incident with $v_{2}$. Thus $G \backslash\left(F_{1} \cup F_{2}\right)$ is a simple graph of order 9 and maximum degree 4 , and is not overfull. Therefore, by Lemma $2 \cdot 1, G \backslash\left(F_{1} \cup F_{2}\right)$ is Class 1 . Therefore $G$ is Class 1 , a contradiction. If $w \neq v_{7}$, then the degree sequence of $\left(G \backslash F_{1}\right) \backslash v^{*}$ is $(2,3,4,4,4,4,4,5)$. The argument is the same, 
except that $F_{2}$ must include an edge incident with $w$ as well, and also leads to a contradiction. In view of (2) and (3), we may assume from now that $|V(G)| \geqslant 11$ and

$$
\Delta(G) \geqslant\left\lceil\frac{2.11}{3}\right\rceil=8
$$

At this point we have shown that either $d_{k}^{\prime} \geqslant k+3(3 \leqslant k \leqslant n-1)$, or $G$ contains a near 1 -factor $F$ which includes the edge $a v_{1}$ and avoids $b$, or $G$ is Class 1 (a contradiction).

Now suppose that either $d_{1}^{\prime} \leqslant 3$ or $d_{2}^{\prime} \leqslant 4$. Then, by $(6), d^{\prime}\left(v^{*}\right)=4$. If $d_{1}^{\prime} \leqslant 3$, then $v^{*}=w_{2}$ and $m_{G}\left(w_{1}, w_{2}\right)=\Delta(G)-3$, contradicting (5). Therefore $d_{1}^{\prime}=4$, so $v^{*}=w_{1}$ and $d^{\prime}\left(w_{2}\right)=4$. If $w_{2} \in\left\{v_{1}, v_{2}\right\}$ then $m_{G}\left(v^{*}, w_{2}\right)=\Delta(G)-3$, again contradicting (5). Therefore $w_{2} \notin\left\{v_{1}, v_{2}\right\}$ and $m_{G}\left(v^{*}, w_{2}\right)=\Delta(G)-4$. Therefore, by (5),

$$
\epsilon(G)=\Delta(G)-6 \quad \text { and } \quad \Delta(G)=|V(G)|-3
$$

and so $d^{*}(v)=2$ for $v \notin\left\{w_{2}, v^{*}\right)$. Therefore $m_{G}\left(v^{*}, v_{1}\right)=m_{G}\left(v^{*}, v_{2}\right)=m_{G}\left(v_{1}, v_{2}\right)=1$ and $m_{G}\left(v^{*}, y\right)=2$.

Let the degrees of $G^{\prime} \mid\left\{v_{1}\right\}$ be $d_{1}^{\prime \prime} \leqslant \ldots \leqslant d_{2 n}^{\prime \prime}$. Then $d_{1}^{\prime \prime}=3, d_{2}^{\prime \prime}=4$,

$$
d_{3}^{\prime \prime}=\ldots=d_{2 n-3}^{\prime \prime}=\Delta(G)-2 \text { and } d_{2 n-2}^{\prime \prime}=d_{2 n-1}^{\prime \prime}=d_{2 n}^{\prime \prime}=\Delta(G)-1 .
$$

Therefore the condition

$$
d_{k}^{\prime \prime} \leqslant k+1 \Rightarrow d_{2 n-1-k}^{\prime \prime} \geqslant 2 n-k \quad(1 \leqslant k \leqslant n-1)
$$

is satisfied, and so, by Lemma $2 \cdot 10, G^{\prime} \backslash\left\{v_{1}\right\}$ has a Hamiltonian circuit containing the edge $a b$. Therefore $G^{\prime} \backslash\left\{v_{1}\right\}$ has a 1 -factor containing $a b$. But $a$ is adjacent in $G^{+}$to $v_{1}$, so $G^{+}$(and therefore $G$ ) has a near 1 -factor $F$ containing the edge $a v_{1}$ and avoiding the vertex $b$.

If $G^{\prime}$ satisfies (7) then $G$ contains a near 1 -factor $F$ including $a v_{1}$ and avoiding $b$. Thus in all cases it follows that either $G$ is Class 1 (a contradiction) or $G$ contains a near 1 -factor $F$ including $a v_{1}$ and avoiding $b$. So from now on we shall assume $G$ contains $F$.

The graph $G \backslash F$ has four vertices, $v^{*}, v_{1}, v_{2}, b$, of maximum degree $\Delta(G)-1$, but the vertex $a$ is adjacent to only one of them $\left(v_{2}\right)$. Therefore, by Lemma 2.6, $G \backslash F$ and $G^{0}=(G \backslash F) \backslash\{a\}_{1}^{\prime}$ have the same chromatic class. The graph $G^{0}$ has three vertices, $v^{*}, v_{1}, b$, of maximum degree, and has $2 n$ vertices.

If $G^{0}$ contains a subgraph $H$ on three vertices with $\Delta\left(G^{0}\right)+1$ edges, then, since $G^{0}$ is a star-multigraph, $v^{*}$ must be one of the vertices. Then $d_{Q^{0}}\left(v^{*}\right)=\Delta\left(G^{0}\right)=\Delta(G)-1$, so $d^{\prime}\left(v^{*}\right) \leqslant 3$. But this contradicts $(6)$. Therefore $G^{0}$ contains no such subgraph.

Since $|V(G)| \geqslant \Delta(G)+3$ it follows that $\left|V\left(G^{0}\right)\right| \geqslant \Delta\left(G^{0}\right)+3$, and so by induction we obtain the contradiction that $G^{0}$ (and therefore $G$ ) is Class 1 , unless $\Delta\left(G^{0}\right)=2 m+1$ for some integer $m, G^{0}$ contains a bridge $e$, and $G^{0} \mid e=G_{1}^{0} \cup G_{2}^{0}$, where $G_{1}^{0} \cap G_{2}^{0}=\varnothing$,

$$
\Delta\left(G_{1}^{0}\right)=2 m+1 \text {, and } G_{1}^{0}=K_{2 m+1}^{*} \quad(\text { for } m \geqslant 2) \text { or } G_{1}^{0}=K_{2 m+1}^{\dagger} \text {. }
$$

The number of edges of $E(G) \backslash E\left(G^{0}\right)$ which are incident with vertices of $G_{1}^{0}$ is at most $2 m+1$. The vertex $v^{*}$ is incident in $G$ with at most one vertex of $V\left(G_{2}^{0}\right) \cup\{a\}$, so at least one of $v_{1}, v_{2}$, say $v_{1}$, is in $V\left(G_{1}^{0}\right)$. But then $v_{1}$ is incident in $G$ with at most two vertices in $V\left(G_{2}^{0}\right) \cup\{a\}$. However, since $d^{*}(v) \geqslant 2(\forall v \in V(G))$, any vertex in $V\left(G_{2}^{0}\right) \cup\{a\}$ is incident with $v^{*}$ or $v_{1}$; consequently $\left|V\left(G_{1}^{0}\right) \cup\{a\}\right| \leqslant 3$. Since all multiple edges of $G$ are incident with $v^{*}$, not more than one edge of $F$ is incident with two vertices of $G_{1}$, and yet each vertex of $G_{1}^{0}$, except for possibly one, is incident with an edge of $F$. 
Therefore $\left|V\left(G_{2}^{0}\right) \cup\{a\}\right| \geqslant\left|V\left(G_{1}^{0}\right)\right|-3=2 m-2$. Therefore $3 \geqslant 2 m-2$, so $m \leqslant 2$, so $\Delta\left(G^{0}\right) \leqslant 5$, and so $\Delta(G) \leqslant 6$. By $(3)$, therefore, $6 \geqslant \frac{2}{3}|V(G)|$, so $9 \geqslant|V(G)|$. However, this contradicts the assumption we are now making that $|V(G)| \geqslant 11$.

Thus there is no critical star multigraph $H$ with three vertices including $v^{*}$ of maximum degree satisfying (2). This proves Theorems 1 and 2.

\section{Proofs of Theorems 3 and 4}

Theorem 3 is an easy consequence of Theorem 1, and Theorem 4 follows from Theorem 3.

Proof of Theorem 3. The cases when $s=1$ or 2 were proved in [3]. Now consider the case when $s=3$. If $n=1$ or 2 , then Theorem 3 is easily seen to be true in this case. So suppose that $n \geqslant 3$. Let $v^{*}$ be the star centre and let $w$ be a vertex which is nonadjacent to $v^{*}$. Then $G \backslash w$ has $2 n+1$ vertices, three vertices of maximum degree, including $v^{*}$, and no induced subgraph on three vertices with more than

$$
\Delta(G \mid w)=2 n-2
$$

edges. Furthermore $G \mid w$ is connected. Therefore $G \mid w$ satisfies the hypotheses of Theorem 1. The minimum degree of $G \mid w$ is $2 n-3=2 n-s \geqslant n$. Therefore $G \mid w$ does not contain a bridge, so $G \backslash w$ does not satisfy Case (1 iii) of Theorem 1. Clearly it does not satisfy Cases (1i) or (1ii) either. Therefore $G \backslash w$ is Class 1 . Since

$$
\begin{aligned}
|E(G \mid w)| & =\frac{1}{2}((2 n+2)(2 n-2))-(2 n-2) \\
& =(2 n-2) n \\
& =\Delta(G \mid w)\left\lfloor\frac{|V(G \mid w)|}{2}\right\rfloor
\end{aligned}
$$

in any edge-colouring of $G \mid w$ with $\Delta(G)=\Delta(G \mid w)$ colours, each colour is missing from exactly one vertex. Therefore any such edge-colouring can be extended to an edgecolouring of $G$ by giving each edge incident with $w$ the colour missing at the corresponding vertex of $G \mid w$. Therefore $G$ is Class 1 . This proves Theorem 3.

Proof of Theorem 4. The sufficiency is trivial, and we need to prove the necessity. Let $G$ be a simple graph with

$$
|V(G)|=2 n+1, \quad \Delta(G)=2 n-2 \quad \text { and } \quad|E(G)|=\Delta(G)\left\lfloor\frac{|V(G)|}{2}\right\rfloor=\left(\begin{array}{c}
2 n+1 \\
2
\end{array}\right)-3 n
$$

Suppose also that $G$ does not contain any overfull subgraph with maximum degree $2 n-2$ (any such subgraph would have $2 n-1$ vertices).

Adjoin a further vertex $v^{*}$ and $2 n-2$ further edges so as to create a regular starmultigraph $G^{*}$ of degrees $2 n-2$ with $2 n+2$ vertices. Since

$$
\left(\begin{array}{c}
2 n+1 \\
2
\end{array}\right)-3 n+(2 n-2)=\frac{1}{2}(2 n+2)(2 n-2),
$$

this can be done. If we show that $G^{*}$ contains no subgraph on three vertices with $2 n-1$ edges, then it follows from Theorem 3 that $G^{*}$ is Class 1 , and therefore $G$ is Class 1 , as required.

Suppose otherwise, namely that $G^{*}$ does have a subgraph on three vertices with $2 n-1$ 
edges. One of the three vertices must be $v^{*}$; let the other two be denoted by $w_{1}$ and $w_{2}$. Then each of the $2 n-2$ edges on $v^{*}$ would have to be joined to $w_{1}$ or $w_{2}$, and $w_{1}$ and $w_{2}$ would have to be joined by one edge. Therefore $w_{1}$ and $w_{2}$ would be joined in $G^{*}$ by a total of $2(2 n-2)-(2 n-2)-2=2 n-4$ edges to vertices of $V\left(G^{*}\right) \backslash\left\{v^{*}, w_{1}, w_{2}\right\}$. Since $G^{*}$ is regular of degree $2 n-2$, it follows that $G^{*} \backslash\left\{v^{*}, w_{1}, w_{2}\right\}$ contains

$$
\frac{1}{2}\{(2 n-1)(2 n-2)-(2 n-4)\}=(2 n-2)(n-1)+1
$$

edges, and so $G^{*} \backslash\left\{v^{*}, w_{1}, w_{2}\right\}$ is overfull, a contradiction.

Therefore $G$ is Class 1 , and Theorem 4 is proved.

The authors started to write this paper (and a number of others to follow) whilst they attended a workshop on latin squares at Simon Fraser University, organised by Dr K. Heinrich. They would like to thank her for inviting them, and also the Natural Sciences and Engineering Research Council of Canada and Simon Fraser University which sponsored the event, and the Open University, England, which provided additional support for the authors.

\section{REFERENCES}

[1] L. W. Berneke and S. Fionini. On small graphs critical with respect to edge-colourings. Discrete Math. 16 (1976), 15-20.

[2] C. BerGe. Graphs and Hypergraphs (North-Holland, 1973).

[3] A. G. Chetwynd and A. J. W. Hnton. Partial edge-colourings of complete graphs or of graphs which are nearly complete. Graph Theory and Combinatorics (Academic Press, vol. in honour of P. Erdös' 70th birthday), 1984, 81-98.

[4] A. G. Cherwynd and A. J. W. Hilton. The chromatic index of graphs of even order with many edges. J. Graph Theory 8 (1984), 463-470.

[5] A. G. Chetwynd and A. J. W. Hinton. Regular graphs of high degree are 1-factorizable. Proc. London Math. Soc. 50 (1985), 193-206.

[6] A. G. Chetwynd and A. J. W. Hinton. Critical star multigraphs. Submitted.

[7] A. G. Chetwynd and A. J. W. Hinton. The edge-chromatic class of graphs with maximum degree at least $|V|-3$. Submitted.

[8] A. G. Chetwynd and A. J. W. Hitron. The edge-chromatic class of graphs of even order with maximum degree at least $|V|-4$. (In preparation.)

[9] A. G. CheTwYND and H. P. YAP. Chromatic index critical graphs of order 9. Discrete Math. 47 (1983), 23-33.

[10] V. ChvÁtal. On Hamilton's ideals. J. Comb. Theory (B), 12 (1972), 163-168.

[11] A. J. W. Hruton and C. A. RodaER. Triangulating nearly complete graphs of odd order. (In preparation.)

[12] I. T. JAKoBSEN. On critical graphs with chromatic index 4. Discrete Math. 9 (1974), 265-276.

[13] M. Plantholt. The chromatic index of graphs with a spanning star. J. Graph Theory 5 (1981), 5-13.

[14] V. G. Vizing. On an estimate of the chromatic class of a $p$-graph [in Russian]. Diskret. Analiz. 3 (1964), 25-30. 